\title{
Melting Temperature of Iron at High Pressure: Statistical Moment Method Approach
}

\author{
Tran Thi Hai ${ }^{1, *}$ Ho Khac Hieu ${ }^{2}$ \\ ${ }^{1}$ Hong Duc University, Thanh Hoa, Vietnam \\ ${ }^{2}$ Duy Tan University, Da Nang, Vietnam
}

Received 08 June 2017

Revised 15 August 2017; Accepted 15 September 2017

\begin{abstract}
The pressure effects on melting temperatures of iron have been studied based on the combination of the modified Lindemann criterion with statistical moment method in quantum statistical mechanics. Numerical calculations have been performed up to pressure $150 \mathrm{GPa}$. Our results are in good and reasonable agreements with available experimental data. This approach gives us a relatively simple method for qualitatively calculating high-pressure melting temperature. Moreover, it can be used to verify future experimental and theoretical works. This research proposes the potential of the combination of statistical moment method and the modified Lindemann criterion on predicting high-pressure melting of materials.
\end{abstract}

Keywords: Melting, High-Pressure, Iron, Moment method.

\section{Introduction}

In recent years, the investigation of melting of materials under high pressure is motivated by the remarkable developments of experimental techniques. Researchers could utilize various methods to measure the melting temperature up to hundreds of GPa [1]. However, up to now, the prediction of high-pressure melting curves of transition metals has been under debate and disagreement among different methods such as diamond-anvil cell experiments [2], X-ray diffraction measurements (XRD) [3], shock-wave experiments [4], computer simulations [5] and theoretical approaches [6]. Consequently, building a theory for determining the melting of materials under high pressure is still the inspiring subjects in physics, especially in geophysics, planet physics, shock physics, and nuclear physics.

In this paper, the melting curve of iron is investigated basing on the Lindemann model which was proposed that [7-9]: Melting of material is going to occur when the ratio between the square root of the mean-square displacement (MSD) and the nearest-neighbor distance (NND) reaches a threshold value. Statistical moment method (SMM) [10] in quantum statistical mechanics will be applied to numerically determine the MSD and NND of iron atoms. Our results are compared with those of previous works up to pressure $150 \mathrm{GPa}$ to verify theoretical approach.

\footnotetext{
*Corresponding author. Tel.: 84-915017980.

Email: tranthihai042016@gmail.com

https//doi.org/ 10.25073/2588-1124/vnumap.4092
} 


\section{Theory}

In order to determine the melting curve of iron at high pressure, the Lindermann's model has been applied with slight modification as the following [11]: We assume that the ratio $\zeta=\sqrt{\left\langle u^{2}\right\rangle} / a$ remains constant for all range of studied pressure. The SMM is used to evaluate MSD $\left\langle u^{2}\right\rangle$ and the NND $a$ between two intermediate atoms under pressure.

Firstly, we summarize the main results of SMM which had been derived for crystalline materials under pressure. From the SMM formalism, the authors have derived the equation of state (EOS) describing the pressure versus volume relation of crystal lattice in the form as [12]

$$
P v=-a\left[\frac{1}{6} \frac{\partial U_{0}}{\partial a}+\frac{\theta X}{2 k} \frac{\partial k}{\partial a}\right], \quad X=\left(\frac{\hbar \omega}{2 \theta}\right) \operatorname{coth}\left(\frac{\hbar \omega}{2 \theta}\right), \theta=k_{B} T,
$$

where $P$ denotes the hydrostatic pressure and $v$ is the atomic volume, $U_{0}$ is the interaction energy of system and $k_{B}$ is the Boltzmann constant. The force constant $k=\left(\frac{\partial^{2} \varphi_{i}}{\partial u_{i x}^{2}}\right)_{e q} \equiv m \omega^{2}$ and anharmonic parameter $\gamma=\frac{1}{6}\left[\left(\frac{\partial^{4} \varphi_{i}}{\partial u_{i x}^{4}}\right)_{e q}+6\left(\frac{\partial^{4} \varphi_{i}}{\partial u_{i x}^{2} \partial u_{i y}^{2}}\right)_{e q}\right]$, where $m$ is the atomic mass and $\varphi_{i}$ is the internal energy associated with atom $i$.

By solving this EOS we can obtain the NND $a P, T$ at pressure $P$ and temperature $T$. For numerical calculations, it is convenient to determine firstly the value of NND at zero temperature. In this case Eq. (1) is reduced to

$$
P v=-a\left[\frac{1}{6} \frac{\partial U_{0}}{\partial a}+\frac{\hbar \omega_{0}}{4 k} \frac{\partial k}{\partial a}\right]
$$

where $\omega_{0}$ is the value of frequency $\omega$ at zero temperature.

For the simplicity, the pair interaction potential between two intermediate atoms is assumed as Lennard-Jones potential type $\varphi r=\varepsilon / m-n\left[n \sigma / a^{m}-m \sigma / a^{n}\right]$, where $\varepsilon$ describes the dissociation energy, $\sigma$ is the equilibrium value of $a$; and the parameters $n$ and $m$ are determined by fitting experimental data (e.g., cohesive energy and elastic modulus). Using this potential we obtain the EOS for crystals at zero temperature as

$$
P v=c_{1}\left(\frac{\sigma}{a}\right)^{m}-c_{2}\left(\frac{\sigma}{a}\right)^{n}+\frac{c_{3}\left(\frac{\sigma}{a}\right)^{n}-c_{4}\left(\frac{\sigma}{a}\right)^{m}}{\sqrt{c_{5}\left(\frac{\sigma}{a}\right)^{n}-c_{6}\left(\frac{\sigma}{a}\right)^{m}}}
$$

where 


$$
\begin{aligned}
& c_{1}=A_{n} \frac{\varepsilon n m}{6 n-m} \quad ; \quad c_{2}=A_{m} \frac{\varepsilon n m}{6 n-m} ; \\
& c_{3}=\frac{1}{a} \frac{\hbar}{4 \sqrt{M}} \sqrt{\frac{\varepsilon n m}{2 n-m}} n+2\left[n+2 A_{n+4}^{a_{i x}^{2}}-A_{n+2}\right] ; \\
& c_{4}=\frac{1}{a} \frac{\hbar}{4 \sqrt{M}} \sqrt{\frac{\varepsilon n m}{2 n-m}}\left[m+2 A_{m+4}^{a_{i x}^{2}}-A_{m+2}\right] ; \\
& c_{5}=n+2 A_{n+4}^{a_{i x}^{2}}-A_{n+2} \quad ; \quad c_{6}=m+2 A_{m+4}^{a_{i x}^{2}}-A_{m+2} .
\end{aligned}
$$

with $A_{n}, A_{m}, A_{n}^{a_{i x}^{2}}, A_{m}^{a_{i x}^{2}}$ are the structural sums of the given crystal [12].

After getting the $\mathrm{NND}_{a} P, 0$, the NND $a P, T$ can be derived

$$
\text { a } P, T=a P, 0+y_{0} P, T,
$$

where $y_{0} P, T$ is the thermally induced lattice expansion which has the form as [12]

$$
\begin{aligned}
& y_{0} P, T=\sqrt{\frac{2 \gamma \theta^{2}}{3 k^{3}} A}, \\
& A=a_{1}+\frac{\gamma^{2} \theta^{2}}{k^{4}} a_{2}+\frac{\gamma^{3} \theta^{3}}{k^{6}} a_{3}+\frac{\gamma^{4} \theta^{4}}{k^{8}} a_{4},
\end{aligned}
$$

and, here, $a_{1}, a_{2}, a_{3}, a_{4}$, and $a_{5}$ were defined as in Ref. 10 .

Using the expression of the second order moment in SMM formalism we obtain the mean-square displacement (MSD) expression as [13]

$$
\left\langle u_{i}^{2}\right\rangle=\left\langle u_{i}\right\rangle^{2}+\theta A_{1}+\frac{\theta}{K} X-1,
$$

where $A_{1}=\frac{1}{K}\left[1+\frac{2 \gamma^{2}}{K^{4}}\left(1+\frac{X}{2}\right) X+1\right]$.

The Lindemann ratio $\zeta P, T$ at pressure $P$ and temperature $T$ now can be determined as

$$
\zeta P, T=\frac{\sqrt{\left\langle u_{i}^{2}\right\rangle}}{a P, T} \text {. }
$$

\section{Results and discussion}

In this section, the expressions derived in the previous section will be used to numerically calculate thermodynamic quantities including the lattice parameter, volume compression and the melting curve of iron at high pressure. The Lennard-Jones potential parameters of iron are $m=3.54, n=6.45, \varepsilon / k_{B}=12576.70 \mathrm{~K}$ and $\sigma=2.4775 \AA$ [14]. 
We firstly determine the Lindemann criterion in within of SMM scheme at experimental melting temperature $T_{m 0}=1811 \mathrm{~K}$ when pressure is zero. It gives the value of Lindemann ratio as $\zeta P=0, T=1811 \mathrm{~K}=0.05256$. This value of Lindemann criterion $\zeta$ is assumed unchanged when pressure increases. It means that, for each pressure $P$, numerical calculations have been performed to derive the temperature $T_{m}$ at which the value of $\zeta P, T_{m}$ is equal to 0.05256 .

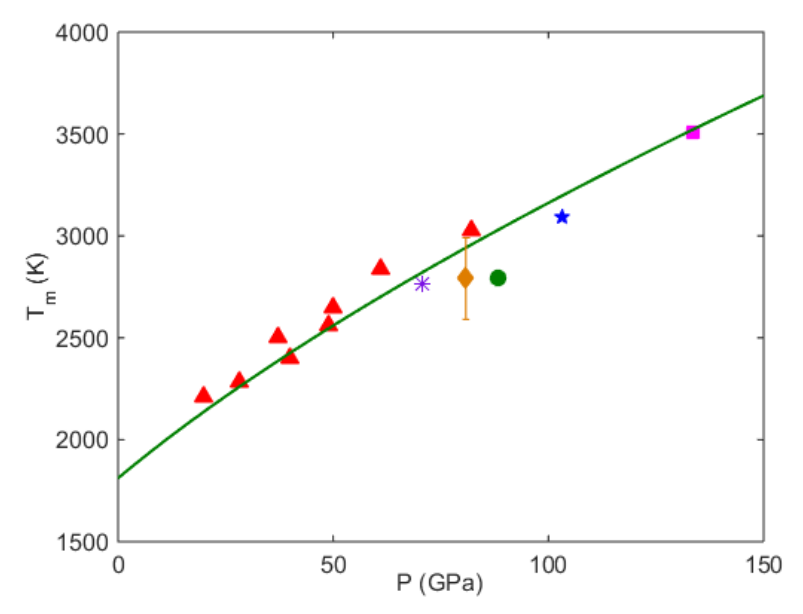

Fig 1. Melting curve of iron. Our results (solid line) are compared with those of shock-compression melting experiments by Ahrens et al. [15] (* mark); the static-compression XRD experiments by Komabayashi and Fei [16] (• mark), Ma et al. [17] (• mark), and Shen et al. [18] ( mark), respectively; in situ XRD measurements by Anzellini et al. ( $\star$ mark) [19]; synchrotron Mössbauer spectroscopy by Jackson et al. [20]( $\Delta$ marks).

In Fig. 1, we show the melting curve of iron in our calculations up to pressure $150 \mathrm{GPa}$ along with the selected recent experimental data of the shock-compression, static-compression, synchrotron Mössbauer spectroscopy and XRD measurements. As it can be seen from this figure, our calculations are reasonable agreement with those of experimental measurements. Especially, recent melting temperature of iron in a laser-heated diamond anvil cell at $T_{m} 103 \mathrm{GPa}=3090 \mathrm{~K}$ obtained by XAS [21] is reasonable consistent with our work $(3195 \mathrm{~K})$. The discrepancy is about $3 \%$. At pressure 135 $\mathrm{GPa}$, our melting evaluation is $T_{m} 135 \mathrm{GPa}=3536 \mathrm{~K}$ while the result of shock-compression melting experiments by Ahrens et al. [15] is $T_{m} 135 \mathrm{GPa}=3400 \pm 200 \mathrm{~K}$. By the onset of convective motion in laser-heated static-compression experiments [22], Boehler derived $T_{m} 135 \mathrm{GPa}=3200 \pm 100 \mathrm{~K}$ and Williams et al. [23] found $T_{m} 135 \mathrm{GPa}=4800 \pm 200 \mathrm{~K}$ by using a combination of static- and shock-compression experiments. Nevertheless, the recent in situ XRD measurements [19] predicted lower melting points in comparison with our calculations. In order to explain such a difference, Anzellini et al. supposed that lower temperatures could be the temperatures of fast recrystallization instead of melting. 


\section{Conclusions}

In this work, we introduced the relatively simple approach to derive the melting curve of iron thanks to the combination of statistical moment method with modified Lindemann criterion of melting. By comparing calculated results with those of available experiments, we conclude that the current approach can be suitable for evaluating the melting of iron up to pressure $150 \mathrm{GPa}$. This approach can also be applied to study the pressure effects on melting temperatures of other metals. It also can be used to verify future multi-anvil and diamond anvil cell experiments, shock-wave experiments as well as theoretical determinations.

\section{Acknowledgments}

This research is funded by the Vietnam National Foundation for Science and Technology Development (NAFOSTED) under grant number 103.01-2017.343.

\section{References}

[1] Y. Mori, H. Ozawa, K. Hirose, R. Sinmyo, S. Tateno, G. Morard, Y. Ohishi, Melting experiments on $\mathrm{Fe}-\mathrm{Fe}{ }_{3} \mathrm{~S}$ system to 254 GPa, Earth Planet. Sci. Lett. 464 (2017) 135-141.

[2] D. Errandonea, High-pressure melting curves of the transition metals $\mathrm{Cu}, \mathrm{Ni}, \mathrm{Pd}$, and Pt, Phys. Rev. B 87 (2013) 054108.

[3] D. Santamaría-Pérez, M. Ross, D. Errandonea, G. D. Mukherjee, M.Mezouar, and R. Boehler, X-ray diffraction measurements of Mo melting to $119 \mathrm{GPa}$ and the high pressure phase diagram, J. Chem. Phys. 130 (2009) 124509.

[4] J. H. Nguyen and N. C. Holmes, Melting of iron at the physical conditions of the Earth's core, Nature 427 (2004) 339.

[5] M. Pozzo and D. Alfè, Melting curve of face-centered-cubic nickel from first-principles calculations, Phys. Rev. B 88 (2013) 024111.

[6] A. B. Belonoshko, L. Burakovsky, S. P. Chen, B. Johansson, A. S.Mikhaylushkin, D. L. Preston, S. I. Simak, and D. C. Swift, Molybdenum at High Pressure and Temperature: Melting from Another Solid Phase, Phys. Rev. Lett. 100 (2008) 135701.

[7] F. Lindemann, The calculation of molecular vibration frequencies, Phys. Z. 11 (1910) 609.

[8] H.K. Hieu, Melting of solids under high pressure, Vacuum. 109 (2014) 184-186.

[9] H. K. Hieu and N. N. Ha, High pressure melting curves of silver, gold and copper, AIP Adv. 3 (2013) 112125.

[10] N. Tang and V. V. Hung, Investigation of the Thermodynamic Properties of Anharmonic Crystals by the Momentum Method. I. General Results for Face-Centred Cubic Crystals, Phys. Status Solid B 149 (1988) 511.

[11] H. K. Hieu, Systematic prediction of high-pressure melting curves of transition metals, J. Appl. Phys. 116 (2014) 163505.

[12] N. Tang and V. V. Hung, Investigation of the Thermodynamic Properties of Anharmonic Crystals by the Momentum Method. III. Thermodynamic Properties of the Crystals at Various Pressures, Phys. Status Solidi B 162 (1990) 371.

[13] H. K. Hieu and V. V. Hung, Study of thermodynamic properties of zinc-blende-type semiconductors: Temperature and pressure dependences, Mod. Phys. Lett. B 25 (2011) 1041.

[14] M. Magomedov, The calculation of the parameters of the Mie-Lennard-Jones potential, High Temp. 44 (2006) 513.

[15] T. J. Ahrens, K. G. Holland,G. Q. Chen. Phase diagram of iron, revised-core temperatures, Geophys. Res. Lett. 29 (2002) 54. 
[16] T. Komabayashi, Y. W. Fei, Internally consistent thermodynamic database for iron to the Earth's core conditions, J. Geophys. Res. Solid Earth 115 (2010) b03202.

[17] Y. Ma, M. Somayazulu, G. Shen, H.-k Mao, J. Shu, R. J. Hemley, In situ X-ray diffraction studies of iron to earth-core conditions, Phys. Earth Planet Inter.143-144 (2004) 455-467.

[18] G. Shen, H.-k. Mao, R. J. Hemley, T. S. Duy, M. L. Rivers, Melting and crystal structure of iron at high pressures and temperatures, Geophys. Res. Lett. 25 (3) (1998) 373-376.

[19] S. Anzellini, A. Dewaele, M. Mezouar, P. Loubeyre, G. Morard, Melting of iron at Earth's inner core boundary based on fast X-ray diffraction, Science 340 (2013) 464-466.

[20] J. M. Jackson, W. Sturhahn, M. Lerche, J. Zhao, T. S. Toellner, E. E. Alp, S. V. Sinogeikin, J. D. Bass, C. A. Murphy, J. K. Wicks, Melting of compressed iron by monitoring atomic dynamics, Earth Planet. Sci. Lett. 362 (2013) 143-150.

[21] G. Aquilanti, A. Trapananti, A. Karandikar, I. Kantor, C. Marini, O. Mathon, S. Pascarelli,R. Boehler, Melting of iron determined by X-ray absorption spectroscopy to 100 GPa, Proc. Natl. Acad. Sci. U.S.A. 112 (2015) 1204212045.

[22] R. Boehler, Temperatures in the Earth's core from melting-point measurements of iron at high static pressures, Nature 363 (1993) 534-536.

[23] Q. Williams, R. Jeanloz, J. Bass, B. Swendsen, T. J. Ahrens, The melting curve of iron to 250 Gigapascals: A constraint on the temperature at Earth's center, Science 236 (1987) 181-182. 